J. DIFFERENTIAL GEOMETRY

84 (2010) 1-17

\title{
A HOPF THEOREM FOR AMBIENT SPACES OF DIMENSIONS HIGHER THAN THREE
}

\author{
Hilário Alencar, Manfredo do Carmo \& Renato Tribuzy
}

\begin{abstract}
We consider surfaces $M^{2}$ immersed in $E_{c}^{n} \times \mathbb{R}$, where $E_{c}^{n}$ is a simply connected $n$-dimensional complete Riemannian manifold with constant sectional curvature $c \neq 0$, and assume that the mean curvature vector of the immersion is parallel in the normal bundle. We consider further a Hopf-type complex quadratic form $Q$ on $M^{2}$, where the complex structure of $M^{2}$ is compatible with the induced metric. It is not hard to check that $Q$ is holomorphic (see [3], p.289). We will use this fact to give a reasonable description of immersed surfaces in $E_{c}^{n} \times \mathbb{R}$ that have parallel mean curvature vector.
\end{abstract}

\section{Introduction}

A beautiful result on surfaces $M^{2}$ immersed in a 3-dimensional euclidean space $\mathbb{R}^{3}$ was obtained by H. Hopf in $1951[6]$ and states that if $M^{2}$ is homeomorphic to a sphere and has constant mean curvature $H$, then $M^{2}$ is totally umbilic, hence isometric to a round sphere. The basic idea of Hopf's proof is to introduce a complex quadratic form $\tilde{\alpha}$ in $M^{2}$ (in the complex structure of $M^{2}$ determined by its induced metric) and prove that $\tilde{\alpha}$ is holomorphic if $H=$ constant. Hopf's theorem was extended by Chern [4] to surfaces immersed in a 3-dimensional Riemannian manifold $M_{c}^{3}$ (we use superscripts to denote dimensions) with constant sectional curvature $c$ and, recently, for surfaces in simplyconnected, homogeneous 3-dimensional Riemannian manifolds with a 4-dimensional group of isometries (Abresch and Rosenberg [1], [2]).

It is then natural to look for higher dimensional ambient spaces in which a Hopf-type theorem holds.

In this paper, we study the case where $x: M^{2} \rightarrow E_{c}^{n} \times \mathbb{R}$ is a surface immersed in the product Riemannian manifold of a simply-connected $n$-dimensional Riemannian manifold $E_{c}^{n}$ of constant sectional curvature $c \neq 0$ with the euclidean line $\mathbb{R}$. We assume that the mean curvature

The authors are partially supported by C.N.Pq.

Received 05/31/2008. 
vector $H$ is parallel in the normal bundle. By analogy with $[\mathbf{1}]$, introduce the real quadratic form in $M^{2}=M$

$$
Q(X, Y)=2\langle\alpha(X, Y), H\rangle-c\langle X, \xi\rangle\langle Y, \xi\rangle,
$$

where $X, Y$ are tangent vectors in $M, \alpha$ is the second fundamental form of $M$ taking values in the normal bundle of $M$, and $\xi$ is the unit tangent vector of $\mathbb{R}$. Let $(u, v)$ be isothermal parameters in $M$, and $z=u+i v$ the corresponding complex parameter. Set

$$
d z=\frac{1}{\sqrt{2}}(d u+i d v), \quad d \bar{z}=\frac{1}{\sqrt{2}}(d u-i d v)
$$

and

$$
Z=\frac{1}{\sqrt{2}}\left(\frac{\partial}{\partial u}-i \frac{\partial}{\partial v}\right), \quad \bar{Z}=\frac{1}{\sqrt{2}}\left(\frac{\partial}{\partial u}+i \frac{\partial}{\partial v}\right) .
$$

In a previous paper [3], we considered this situation and proved that the (2,0)-part $Q^{(2,0)}=\psi d z d z$ of $Q$ is holomorphic. This means that the complex function

$$
Q^{(2,0)}(Z, Z)=2\langle\alpha(Z, Z), H\rangle-c\langle\xi, Z\rangle^{2}=\psi
$$

is holomorphic.

In the present paper, we want to use the fact that $Q^{(2,0)}$ is holomorphic to give a reasonable description of which immersed surfaces in $E_{c}^{n} \times \mathbb{R}$ have parallel mean curvature vector. All manifolds are connected and oriented.

Our first result shows, under no global hypothesis on $M$, that either $H$ is an umbilic direction, and our surface is entirely contained in $E_{c}^{n}$ (this reduces our question to a theorem of S.T. Yau; see items (1) and (2) below) or we can reduce the codimension of our immersion to three.

Theorem 1. Let $M$ be a surface and $E_{c}^{n}$ be a Riemannian manifold of constant sectional curvature $c \neq 0$, and let $x: M \rightarrow E_{c}^{n} \times \mathbb{R}$ be an immersion with parallel mean curvature vector. Then, one of the following assertions holds:

1) $x(M)$ is a minimal surface in a totally umbilical hypersurface of $E_{c}^{n}$.

2) $x(M)$ is a surface with constant mean curvature of a 3-dimensional totally umbilic or totally geodesic submanifold of $E_{c}^{n}$.

3) $x(M)$ lies in $E_{c}^{4} \times \mathbb{R}$.

To improve the last part of the above result, we add further hypotheses on $M$; this is the subject of the following two theorems.

Theorem 2 is where we can be most precise by assuming that $M$ is homeomorphic to a sphere. We will show that either $H$ is an umbilic direction, and we are in the same situation as items (1) and (2) in Theorem 1, or we are in the situation of item (4) below which generalizes the result of Abresch and Rosenberg [1] to immersions of spheres in $E_{c}^{n} \times \mathbb{R}$. The proof of (4) is delicate and is the main point of Theorem 2 . 
Theorem 2. Let $M$ be a compact surface of genus zero and let $x: M \rightarrow E_{c}^{n} \times \mathbb{R}$ be an immersion of $M$ with parallel mean curvature. Then, one of the following assertions holds:

1) $x(M)$ is a minimal surface of a totally umbilical hypersurface of $E_{c}^{n}$.

2) $x(M)$ is a standard sphere of a totally umbilical 3-dimensional submanifold of $E_{c}^{n}$.

3) $x(M)$ is a standard sphere of $E_{c}^{3}$.

4) $x(M)$ lies in $E_{c}^{4} \times \mathbb{R} \subset \mathbb{R}^{6}$ (possiby with the Lorentz metric), and there exists a plane $P$ such that $x(M)$ is invariant for rotations which fix its orthogonal complement. Furthermore, the level curves of the height function $p \mapsto\langle x(p), \xi\rangle$ are circles lying in planes parallel to $P$.

For the next theorem, we assume that $M$ is complete, that its Gaussian curvature $K \geq 0$, and that $c<0$. Even for $n=2$, Theorem 3 seems to be new.

Theorem 3. Let $M$ be a complete surface with Gaussian curvature $K \geq 0$, and let $x: M \rightarrow E_{c}^{n} \times \mathbb{R}, c<0$, be an isometric immersion with parallel mean curvature. Then one of the following assertions holds:

1) $K \equiv 0$.

2) $x(M)$ is a minimal surface of a totally umbilical hypersurface of $E_{c}^{n}$.

3) $x(M)$ is a surface with constant mean curvature in a totally umbilical 3-dimensional submanifold of $E_{c}^{n}$.

4) $x(M)$ lies in $E_{c}^{4} \times \mathbb{R} \subset \mathbb{R}^{6}$ (with the Lorentz metric), and there exists a plane $P$ such that the level lines of the height function $p \mapsto$ $\langle x(p), \xi\rangle$ are curves lying in planes parallel to $P$.

Remark 1. Because the mean curvature vector of our immersion is parallel, the immersion itself is analytic; this means that the functions of two real variables that define locally the map $x: M^{2} \rightarrow E_{c}^{n} \times \mathbb{R}$ are real analytic functions (See, C.B. Morrey Jr. On the analiticity of solutions of analytic nonlinear elliptic systems of partial differential equations, American J. of Math. 80 (1958), 198-237). Such function satisfy a principle of analytic continuation (See J. Dieudonné, Foundations of Modern Analysis, Academic Press, 1969, Chapter IX (2.4.2)) which has the following consequence: Let $V$ be an open, connected subset of $R^{n}$ and let $f: V \rightarrow R^{k}$ be a real analytic map in $V$. Let $U \subset V$ be an open subset of $R^{n}$. If $f \equiv 0$ in $U$ then $f \equiv 0$ in $V$. Thus an analytic map cannot vanish in any open set unless it is identically zero.

Remark 2. We should mention that holomorphic quadratic forms on surfaces that lie in products of space forms of arbitrary dimensions have been found in the Doctor's thesis of F.M.A. Vitrio (Fortaleza 2007) and will appear in the joint work of J.H. de Lira and F.M.A. Vitrio: 
Isometric immersions into Riemannian products, preprint. As far as we know, there is no overlapping with the present paper.

Remark 3. An interesting question is to characterize those surfaces for which $K \equiv 0$ in Theorem 3 . This seems to be unknown even for the case of a complete $M^{2}$ immersed in $M_{c}^{2} \times \mathbb{R}, \quad c \neq 0$.

Acknowledgement. We want to thank Harold Rosenberg for conversations during the preparation of this paper.

\section{Proof of Theorem 1}

We will start with a few lemmas.

Lemma 0. Let $x: M \rightarrow E_{c}^{n} \times \mathbb{R}$ be an immersion of a surface. Assume that a subbundle $L$ of the normal bundle contains the image of the second fundamental form, is parallel in the normal connection and that $T M \oplus L=V$ is invariant by the curvature tensor $\widetilde{R}$ of $E_{c}^{n} \times \mathbb{R}$ in the following sense: Whenever $A, B, C \in V$, we have

$$
\widetilde{R}(A, B) C \in V .
$$

Then, there exists a totally geodesic submanifold $S \subset E_{c}^{n} \times \mathbb{R}$, with $T_{p} S=V$ for all $p \in S$, so that $x(M) \subset S$.

Proof. This follows from ([5], Theorem 2), since $E_{c}^{n} \times \mathbb{R}$, with the LeviCivitta connection of the product metric, is a reductive homogeneous space.

q.e.d.

Remark 4. We recall that for any bundle $E \rightarrow B$ with a connection $\nabla$, we say that a subbundle $E^{\prime} \rightarrow B, E^{\prime} \subset E$, is parallel if $E^{\prime}$ is invariant by the connection $\nabla$.

Lemma 1. Let $x: M \rightarrow E_{c}^{n} \times \mathbb{R}$ be an immersion of a surface $M$ with parallel mean curvature. Then for all $v \in T^{\perp} M, A_{H}$ commutes with $A_{v}$; here $A_{v}$ is the second fundamental form (as a linear map in $T M)$ corresponding to the normal vector $v$, namely, $\left\langle A_{v}(X), Y\right\rangle=$ $\langle\alpha(X, Y), v\rangle$.

Proof. By the Ricci equation,

$$
\left\langle R^{\perp}(X, Y) H, v\right\rangle=\left\langle\left[A_{H}, A_{v}\right] X, Y\right\rangle+\langle\widetilde{R}(X, Y) H, v\rangle,
$$

where $\widetilde{R}$ is the curvature tensor of $E_{c}^{n} \times \mathbb{R}$, and $R^{\perp}$ the curvature tensor of the normal bundle of the immersion. Since $H$ is parallel in the normal bundle, by the definition of $R^{\perp}, R^{\perp}(X, Y) H=0$. Thus, it suffices to show that $\langle\widetilde{R}(X, Y) H, v\rangle=0$.

Let $A, B, C, D$ be orthogonal vectors of $E_{c}^{n} \times \mathbb{R}$ and denote by $A_{2}$ the projection of $A$ onto the second factor, namely $A_{2}=\langle A, \xi\rangle \xi$, and 
the same for the other vectors. Then, since $\pi A=A-A_{2}$, where $\pi$ is the projection onto the tangent space of $E_{c}^{n}$, we obtain

$$
\begin{aligned}
\langle\widetilde{R}(A, B) C, D\rangle= & c\{\langle\pi A, \pi C\rangle\langle\pi B, \pi D\rangle-\langle\pi A, \pi D\rangle\langle\pi B, \pi C\rangle\} \\
= & c\left\{\left(\langle A, C\rangle-\left\langle A_{2}, C_{2}\right\rangle\right)\left(\langle B, D\rangle-\left\langle B_{2}, D_{2}\right\rangle\right)\right. \\
& \left.-\left(\langle A, D\rangle-\left\langle A_{2}, D_{2}\right\rangle\right)\left(\langle B, C\rangle-\left\langle B_{2}, C_{2}\right\rangle\right)\right\},
\end{aligned}
$$

where we have used that $\left\langle A_{2}, C\right\rangle=\left\langle A_{2}, C_{2}\right\rangle=\left\langle A, C_{2}\right\rangle$, and similarly for the other vectors.

We need to exclude the case where $\xi \in T_{p} M$, for all $p \in M$. For that, we have the following

Sublemma. If $\xi \in T_{p} M$, for all $p \in T_{p} M$, then $x(M) \subset E_{c}^{2} \times \mathbb{R}$. This case has already been included in item (3) of Theorem 1. Actually, in this case $x(M)$ is a vertical cylinder over a curve in $E_{c}^{2}$ of geodesic curvature $2 \mathrm{H}$.

Proof of the Sublemma. Since $\xi \in T_{p} M$, for all $p \in M$, we can choose a basis $\left\{e_{1}, e_{2}\right\}$ for $T M$ by setting $e_{1}=\xi$ and $e_{2}$ to be a unit vector orthogonal to $e_{1}$ so that $\left\{e_{1}, e_{2}\right\}$ is a positive basis.

We first show that $\alpha\left(e_{1}, e_{2}\right)=0$. To see that let $\left\{e_{\beta}\right\}, \beta=3, \ldots, n+1$, be an orthonormal basis of $T M^{\perp}$. Then, for all $\beta$,

$$
\left\langle\alpha\left(e_{1}, e_{2}\right), e_{\beta}\right\rangle=\left\langle\alpha\left(e_{2}, e_{1}\right), e_{\beta}\right\rangle=\left\langle\tilde{\nabla}_{e_{2}} e_{1}, e_{\beta}\right\rangle-\left\langle\nabla_{e_{2}} e_{1}, e_{\beta}\right\rangle=0,
$$

where we have used that $e_{1}$ is parallel in the conexion $\tilde{\nabla}$ of the ambient space. Since $\alpha\left(e_{1}, e_{2}\right)$ is a normal vector, it follows from the above that $\alpha\left(e_{1}, e_{2}\right)=0$. In a similar way, it can be shown that $\alpha\left(e_{1}, e_{1}\right)=0$. Thus

$$
\alpha\left(e_{1}, e_{1}\right)+\alpha\left(e_{2}, e_{2}\right)=\alpha\left(e_{2}, e_{2}\right)=2 H,
$$

and, since $H$ is parallel in the normal conexion, so is $\alpha\left(e_{2}, e_{2}\right)$. It follows that the first normal space of the immersion, i.e., the image of the second fundamental form, is a 1-dimensional parallel subspace of the normal space.

We now set $\bar{L}=\operatorname{Im}(\alpha)$. Since we know that $\bar{L}$ is parallel in the normal bundle, we have that $\bar{L} \oplus T M=\bar{V}$ is parallel in the connection $\widetilde{\nabla}$. Choose an orthonormal basis for $\bar{V}$ so that $\xi$ belongs to that basis. We want to show that $\bar{V}$ is invariant by $\widetilde{R}$. By linearity, we have only to check the invariance of $\widetilde{R}$ for elements in that basis, say, $\left\{a_{1}, a_{2}, a_{3}\right\}$. Let $\eta \in \bar{V}^{\perp}$. It follows from Eq. (1) that for all $i, j, k=1,2,3$, we have

$$
\left\langle\widetilde{R}\left(a_{i}, a_{j}\right) a_{k}, \eta\right\rangle=0, \text { for all } \eta \in \bar{V}^{\perp} .
$$

Thus $\widetilde{R}\left(a_{i}, a_{j}\right) a_{k} \in \bar{V}$, i.e., $\bar{V}$ is invariant by $\widetilde{R}$. Using Lemma 0 , and the fact that $\operatorname{dim} \bar{L}=1$, we conclude that the codimension reduces to one. Furthermore, since $\xi \in \bar{V}$, the totally geodesic subspace $S$ of Lemma 0 is $E_{c}^{2} \times \mathbb{R}$ and the Sublemma is proved. In addition, since actually $\xi \in T M$, the last sentence of the Sublemma is obvious. 
Remark 4. Let $\varphi: M \rightarrow \mathcal{L}\left(T M^{\perp}, \mathbb{R}\right)$ be the map that takes $p \in M$ to the linear function $\varphi_{p}$ of $T_{p} M$ given by

$$
\varphi_{p}\left(\eta_{p}\right)=\left\langle\eta_{p}, \xi\right\rangle
$$

where $\eta_{p}$ is a normal vector at $p$. Notice that $\xi \in T_{p} M$ iff $\varphi_{p}$ vanishes for all $\eta_{p}$. By analyticity, either $\varphi$ is identically zero or the set of zeroes of $\varphi$ is a closed set $T$ with no interior points. Thus, either $\xi \in T_{p} M$ everywhere, or $\xi \in T_{p} M$ in $T$. In the first case, the codimension reduces to one (See the Sublemma above) and the situation is well known. We will handle the second case in what follows.

Let us return to the proof of Lemma 1 .

Proof of the Lemma 1. Let us introduce a basis in $T^{\perp} M$. Let $u$ be the projection of $\xi$ in the normal bundle and set $e_{3}=\frac{u}{|u|}$. This is well defined only in the complement $\mathcal{C} T$ of the set $T$ defined in Remark 4 . Notice that $\mathcal{C} T$ is an open and dense set in $M$. Complete $e_{3}$ into an orthonormal basis of $T^{\perp} M,\left\{e_{3}, e_{4}, \ldots, e_{n-1}\right\}$.

Now let $\left\{e_{1}, e_{2}\right\}$ be an orthonormal basis for $T M$ and let us agree in the following set of indices: latin letters $i, j, k$, etc. will vary in 1,2 and greek letters $\alpha, \beta$, etc. will vary in $3,4, \ldots, n+1$. It follows from equation (1) that

$$
\left\langle\widetilde{R}\left(e_{i}, e_{j}\right) e_{\alpha}, e_{\beta}\right\rangle=0,
$$

if $\alpha$ or $\beta$ is distinct from 3, since in this case, $\left\langle e_{\alpha}, \xi\right\rangle=0$ or $\left\langle e_{\beta}, \xi\right\rangle=0$. Furthermore, $\left\langle\widetilde{R}\left(e_{i}, e_{j}\right) e_{3}, e_{3}\right\rangle=0$. It follows that $(\widetilde{R}(X, Y) v)^{\perp}=0$ and Lemma 1 is proved in $\mathcal{C} T$. Since $\mathcal{C} T$ is open and dense, by continuity, the equality $(\widetilde{R}(X, Y) v)^{\perp}=0$, hence Lemma 1 , holds everywhere in $M$.

q.e.d.

Corollary of the proof. Either there exists a basis that diagonalizes $A_{v}$, for all $v \in T^{\perp} M$ or $A_{H}$ is a multiple of the identity, that is, $H$ is an umbilic direction.

The crucial step in the proof of Theorem 1 is the following Lemma.

Lemma 2. If $H$ is nowhere an umbilic direction, then there exists a subbundle of the normal bundle that is parallel, contains the image of the second fundamental form $\alpha$ and has dimension $\leq 3$.

Proof. Set

$$
L=\operatorname{span}\left\{\operatorname{Im} \alpha \cup e_{3}\right\} .
$$

We recall that $e_{3}$ is the unit vector of the projection of $\xi$ in the normal space and it is only well defined in the complement $\mathcal{C} T$ of the set $T$ defined in Remark 4 . So, for the time being, we restrict ourselves to $\mathcal{C} T$.

We will show that $L$ is parallel and this will prove the Lemma. 
It suffices to show that if a normal subbundle $W \perp L$ then $\nabla^{\perp} W \perp L$. By Lemma 1, there exists a frame, say $\left\{e_{1}, e_{2}\right\}$, that diagonalizes $\alpha$. Let $w \in W$. We first show that

$$
-A_{i j k}=\left\langle\alpha\left(e_{i}, e_{j}\right), \nabla_{e_{k}}^{\perp} w\right\rangle=0, \text { for all } i, j, k=1,2 .
$$

Indeed, since $w \perp L$,

$$
\left\langle\alpha\left(e_{i}, e_{j}\right), \nabla_{e_{k}}^{\perp} w\right\rangle=-\left\langle\nabla_{e_{k}}^{\perp} \alpha\left(e_{i}, e_{j}\right), w\right\rangle=A_{i j k} .
$$

Since $\alpha$ is symmetric, $A_{i j k}=A_{j i k}$. Furthermore, by definition,

$$
\begin{aligned}
& \left\langle\left(\nabla_{e_{k}}^{\perp} \alpha\right)\left(e_{i}, e_{j}\right), w\right\rangle=\left\langle\nabla_{e_{k}}^{\perp} \alpha\left(e_{i}, e_{j}\right), w\right\rangle-\left\langle\alpha\left(\nabla_{e_{k}} e_{i}, e_{j}\right), w\right\rangle \\
& -\left\langle\alpha\left(e_{k}, \nabla_{k} e_{j}\right), w\right\rangle=\left\langle\nabla_{e_{k}}^{\perp} \alpha\left(e_{i}, e_{j}\right), w\right\rangle,
\end{aligned}
$$

since $w \perp \operatorname{Im} \alpha$. By using the equation of Codazzi,

$$
\begin{aligned}
\left(\nabla_{e_{k}}^{\perp} \alpha\right)\left(e_{i}, e_{j}\right) & =\left(\nabla_{e_{i}}^{\perp} \alpha\right)\left(e_{k}, e_{j}\right)+\left(\widetilde{R}\left(e_{k}, e_{i}\right) e_{j}\right)^{\perp} \\
& =\left(\nabla_{e_{j}}^{\perp} \alpha\right)\left(e_{i}, e_{k}\right)+\left(\widetilde{R}\left(e_{i}, e_{j}\right) e_{k}\right)^{\perp} .
\end{aligned}
$$

But, by $\left.(1),\left\langle\widetilde{R}\left(e_{k}, e_{i}\right) e_{j}, w\right\rangle=0=\left\langle\widetilde{R}\left(e_{i}, e_{j}\right) e_{k}\right), w\right\rangle$. Thus,

$$
A_{i j k}=A_{k j i}=A_{i k j} \text {. }
$$

Notice now that

$$
\left\langle\alpha\left(e_{i}, e_{j}\right), \nabla_{e_{k}}^{\perp} w\right\rangle=\left\langle A_{\nabla_{e_{k}} w} e_{i}, e_{j}\right\rangle .
$$

So, since $\left\{e_{i}, e_{j}\right\}$ diagonalizes $\alpha$, if $i \neq j,\left\langle\alpha\left(e_{i}, e_{j}\right), \nabla_{e_{k}}^{\perp} w\right\rangle=-A_{i j k}=0$, $i \neq j$. It follows from the above and (3) that if two of the indices $i$, $j, k$ are distinct, $A_{i j k}=0$. We now show that if all indices are equal, $A_{i i i}=0$. Indeed,

$$
\begin{aligned}
& A_{i i i}=\left\langle\nabla_{e_{i}}^{\perp} \alpha\left(e_{i}, e_{i}\right), w\right\rangle=-\left\langle\alpha\left(e_{i}, e_{i}\right) \nabla_{e_{i}}^{\perp} w\right\rangle \\
- & \left\langle\alpha\left(e_{j}, e_{j}\right), \nabla_{e_{i}}^{\perp} w\right\rangle+\left\langle\alpha\left(e_{j}, e_{j}\right), \nabla_{e_{i}}^{\perp} w\right\rangle \\
- & \left\langle 2 H, \nabla_{e_{i}}^{\perp} w\right\rangle+\left\langle\alpha\left(e_{j}, e_{j}\right), \nabla_{e_{i}}^{\perp} w\right\rangle=2\left\langle\nabla_{e_{i}}^{\perp} H, w\right\rangle+A_{j j i}=0 .
\end{aligned}
$$

This proves (2). It remains to prove that if $w \in W$, then $\nabla_{e_{k}} w \perp e_{3}$. For that, observe that, being a product of locally symmetric spaces, $E_{c}^{n} \times \mathbb{R}$ is locally symmetric. This means that

$$
\left(\widetilde{\nabla}_{Z} \widetilde{R}\right)(X, Y, w)=0, \quad \text { for all } X, Y, Z \text { tangent vectors. }
$$

But

$$
\begin{array}{r}
\left(\widetilde{\nabla}{ }_{Z} \widetilde{R}\right)(X, Y, w)=Z \widetilde{R}(X, Y, w)-\widetilde{R}\left(\nabla_{Z} X, Y, w\right)-\widetilde{R}(\alpha(X, Z), Y, w) \\
-\widetilde{R}(X, \alpha(Y, Z), w)-\widetilde{R}\left\langle X, \nabla_{Z} Y, w\right)+\widetilde{R}\left(X, Y, A_{w} Z\right)-\widetilde{R}\left(X, Y, \nabla_{Z}^{\perp} w\right) .
\end{array}
$$

Observe that, by Eq. (1) if $C$ is orthogonal to $A$ and $B$, and $C_{2}=$ $\langle C, \xi\rangle=0$, then $\widetilde{R}(A, B) C=0$. Thus all terms of the right hand side of the above equality vanish, except for the two last ones. Furthermore, since $w \perp \operatorname{Im}(\alpha)$, we have that $A_{w}=0$. It follows that $\widetilde{R}\left(X, Y, \nabla \frac{\perp}{Z} w\right)=$ 0 , for all $X, Y, Z$, with $X \perp Y$. 
We now assume that $X_{2} \neq 0$ and $Y_{2}=0$, then, by Eq. (1) it follows that

$$
\left\langle\widetilde{R}(X, Y) \nabla_{Z}^{\perp} w, Y\right\rangle=\langle X, \xi\rangle\left\langle\nabla \frac{\perp}{Z} w, \xi\right\rangle=0 .
$$

Therefore $\left\langle\nabla \frac{1}{Z} w, \xi\right\rangle=0$, and this proves Lemma 2, for $\mathcal{C} T \subset M$. Since $\mathcal{C} T$ is open and dense in $M$, the normal bundle $W$ is still parallel throughout $M$, by continuity. This proves that Lemma 2 holds in $M$.

q.e.d.

In case that $H$ is an umbilic direction, we need the following Lemma. We recall that we proved in $([\mathbf{3}]$ Remark 2.3$)$, that the $(2,0)$-part $Q^{(2,0)}$ of $Q$ given by

$$
Q(X, Y)=2\langle\alpha(X, Y), H\rangle-c\langle X, \xi\rangle\langle Y, \xi\rangle
$$

is holomorphic.

Lemma 3. Let $H$ be nonzero and an umbilic direction everywhere. Then $\langle X, \xi\rangle=0$, for every tangent vector field $X$. It follows that $T M \subset$ $T E_{c}^{n}$, hence $x(M) \subset E_{c}^{n}$.

Proof. By definition of $Q$, we can write $Q^{(2,0)}=Q(Z, Z) d z^{2}$ where

$$
Q(Z, Z)=2\langle\alpha(Z, Z), H\rangle-c\langle\xi, Z\rangle^{2} .
$$

Now choose an orthonormal basis $\{X, Y\}$ for $T M$. Then

$$
\langle\alpha(Z, Z), H\rangle=\langle\alpha(X, X)-\alpha(Y, Y)-2 i \alpha(X, Y), H\rangle .
$$

Since $H$ is an umbilic direction, $A_{H} Z=\mu Z$, for $Z \in T M$. Thus

$$
\langle\alpha(X, X), H\rangle=\left\langle A_{H} X, X\right\rangle=\mu|X|^{2}=\mu=\langle\alpha(Y, Y\rangle, H\rangle
$$

and

$$
\langle\alpha(X, Y), H\rangle=0 .
$$

It follows that if $H$ is an umbilic direction,

$$
Q(Z, Z)=-c\langle\xi, Z\rangle^{2} .
$$

Furthermore, because $Q^{(2,0)}$ is holomorphic,

$$
\begin{aligned}
0=\bar{Z} Q(Z, Z)=-c \bar{Z}\langle Z, \xi\rangle^{2} & =-2 c\langle Z, \xi\rangle\left\langle\widetilde{\nabla}_{\bar{Z}} Z, \xi\right\rangle \\
& =-2 c\langle\alpha(\bar{Z}, Z), \xi\rangle\langle\xi, Z\rangle
\end{aligned}
$$

since $\left(\widetilde{\nabla}_{\bar{Z}} Z\right)^{T}=0$ and, by definition,

$$
\alpha(Z, \bar{Z})=\widetilde{\nabla}_{\bar{Z}} Z-\left(\widetilde{\nabla}_{\bar{Z}} Z\right)^{T}=\left(\widetilde{\nabla}_{\bar{Z}} Z\right)^{N} .
$$


Notice that, by denoting $e_{1}$ and $e_{2}$ the unit vectors of $\frac{\partial}{\partial u}, \frac{\partial}{\partial v}$, respectively, we have

$$
\begin{aligned}
\alpha(Z, \bar{Z}) & =\lambda^{2} \alpha\left(\frac{e_{1}-i e_{2}}{\sqrt{2}}, \frac{e_{1}+i e_{2}}{\sqrt{2}}\right) \\
& =\frac{\lambda^{2}}{2}\left\{\alpha\left(e_{1}, e_{1}\right)+\alpha\left(e_{2}, e_{2}\right)\right\}=\lambda^{2} H .
\end{aligned}
$$

Thus, from

$$
0=-2 c\langle\alpha(\bar{Z}, Z), \xi\rangle\langle\xi, Z\rangle=-2 c \lambda^{2}\langle H, \xi\rangle\langle\xi, Z\rangle
$$

we obtain that either $\langle\xi, Z\rangle=0$ or $\langle H, \xi\rangle=0$. Let $G$ be the set of zeroes of $\langle\xi, Z\rangle$. $G$ is not $M$; otherwise, $\left\langle\xi, e_{1}\right\rangle=\left\langle\xi, e_{2}\right\rangle=0$ and we are done. By analyticity, $G$ is a closed set with no interior points. In the open and dense complement $\mathcal{C} G$ of $G$, we have that $\langle H, \xi\rangle=0$. Thus $X\langle H, \xi\rangle=0$, for all $X$. Since $H$ is parallel in the normal connection and $\xi$ is parallel, we have

$$
0=X\langle H, \xi\rangle=\left\langle\widetilde{\nabla}_{X} H, \xi\right\rangle+\left\langle H, \widetilde{\nabla}_{X} \xi\right\rangle=-\left\langle A_{H} X, \xi\right\rangle .
$$

But $A_{H}$ is a nonzero multiple of the identity. Thus $\langle X, \xi\rangle=0$, for all $X$ and all points in $\mathcal{C} G$. By the continuity of $\langle X, \xi\rangle$ in $M$, this holds for the whole $M$. This proves Lemma 3.

q.e.d.

Remark 5. Notice that the map $p \mapsto\left(A_{H}-\mu I\right)(p), \mu$ a constant, is analytic. If $H$ is an umbilic direction (which is a zero of the above map), either this holds everywhere in $M$ or it holds for a closed set without interior points in $M$. The first case is treated by Lemma 3. For the second case, $H$ is not an umbilic direction in an open, dense set $W$ in $M$. In the proof of Lemma 2, we proved that it holds throughout $W$. By continuity it holds in $M$. Thus, Lemmas 2 and 3 exhaust all possible situations, and the final conclusion is:

Either $H$ is everywhere an umbilic direction, and $M^{2} \subset E_{c}^{n}$, or $H$ is nowhere an umbilic direction, and there exists a subbundle of the normal bundle that is parallel, contains the image of the second fundamental form and has dimension greater or equal to three.

We now need a theorem of reduction of codimension.

Theorem A. Let $x: M \rightarrow E_{c}^{n} \times \mathbb{R}$ be an immersed surface, assume that $H$ is nowhere an umbilic direction, and let $L=\operatorname{Span}\left\{\operatorname{Im}(\alpha), e_{3}\right\}$. Set $V=L \oplus T M$. Then there exists a totally geodesic submanifold $S \subset E_{c}^{n} \times \mathbb{R}$, with $\operatorname{dim} S=\operatorname{dim} V$, such that $x(M) \subset S$.

Proof. If follows from Lemma 2 that the bundle $L \oplus T M$ is parallel in the connection $\widetilde{\nabla}$. Further, $V$ is invariant by $\widetilde{R}$ in the sense of Lemma 0 . This follows from Eq. (1) by the same argument we used in the proof of the Sublemma of Lemma 1.

We now apply Lemma 0, and this proves the Theorem A. q.e.d. 
We also need a theorem of Yau [8]:

Theorem B (Yau, Theorem 4). Let $x: M^{2} \rightarrow E_{c}^{n}$ a surface immersed in $E_{c}^{n}$ with parallel mean curvature vector. Then, either $M^{2}$ is a minimal surface of an umbilical hypersurface of $E_{c}^{n}$ or $M^{2}$ lies in a 3-dimensional umbilical submanifold of $E_{c}^{n}$ with constant mean curvature.

We now complete the proof of Theorem 1 . If $H$ is an umbilic direction, by Lemma $3, M \subset E_{c}^{n}$, and by Theorem B, we obtain items (1) and (2). If $H$ is not an umbilic direction, there exists a normal subbundle $L$ that is parallel in the normal connection, contains the image of $\alpha$ and $\operatorname{dim} L \leq 3$ (Lemma 2). By Theorem A, $M$ is actually contained in a totally geodesic submanifold $S$ of $E_{c}^{n}(c) \times \mathbb{R}$ of dimension at most five. Since the set $V$ of Theorem A contains a nonzero multiple of $\xi, S$ is actually of the form $E_{c}^{4} \times \mathbb{R}$. Thus, we obtain (3) of Theorem 1. This concludes the proof of Theorem 1 .

\section{Proof of Theorem 2}

For the purpose of our future computations, we want to consider the following cases:

Case a. Assume that $\xi \perp T_{p} M$, for all $p$, in an open, connected subset of $M$. Then this open subset lies in $E_{c}^{n}$. By analyticity, the same holds for $x(M)$. As we have seen before (Lemma 3 of the previous Section), this occurs if $H$ is an umbilic direction and implies that $x(M) \subset E_{c}^{n}$. In the previous Section, this case was handled by using Yau's theorem [8].

Case b. $\xi \in T_{p} M$, for all $p$ in an open, connected subset of $M$. It can be shown in that case that the first normal space is parallel and has dimension one (See Sublemma of Section 2). It follows that the above open subset lies in $E_{c}^{2} \times \mathbb{R}$. By analyticity, the same holds for $x(M)$. However, under the hypothesis of Theorem 2, we have the following

Assertion. If $M$ is homeomorphic to a sphere, Case b cannot occur.

Proof of the Assertion.

The proof is very simple. Since $\xi$ is a parallel vector field, the hypothesis in Case b means that we have a parallel vector in a connected, open subset $U$ of $M$. This implies that the Gaussian curvature $K$ vanishes in $U$ and, by analyticity, everywhere in $M$. But, by the Gauss-Bonnet formula, the integral of $K$ in $M$ is positive. Hence, there are points where $K$ is positive, a contradiction. This proves the Assertion.

Since Case a can be handled by using Yau's theorem as in Theorem 1, and this will take care of items 1 to 3 , of Theorem 2, we will assume 
that we are not in Case a and will concentrate ourselves in the proof of item 4. Notice that in our situation, $H$ is not an umbilic direction.

Choose an orthonormal frame $\left\{e_{1}, e_{2}, \ldots, e_{n+1}\right\}$ such that $e_{1}, e_{2}$ are tangent vectors, and $e_{1}$ and $e_{3}$ are the unit vectors in the directions of the projections of $\xi$ in the tangent and normal spaces, respectively; this means that $\left\langle\xi, e_{A}\right\rangle=0$, if $A$ is distinct from 1 and 3 .

Since $M^{2}$ is homeomorphic to a sphere, the holomorphic function $Q^{(2,0)}(Z, Z)$ vanishes. Then we obtain that

$$
Q\left(e_{1}, e_{1}\right)=Q\left(e_{2}, e_{2}\right), \quad Q\left(e_{1}, e_{2}\right)=0 .
$$

Therefore, $2\left\langle\alpha\left(e_{1}, e_{2}\right), H\right\rangle=c\left\langle\xi, e_{1}\right\rangle\left\langle\xi, e_{2}\right\rangle$.

Since $\left\langle\xi, e_{2}\right\rangle=0$, the basis $\left\{e_{1}, e_{2}\right\}$ diagonalizes $A_{H}$, hence, by Lemma 1 , diagonalizes $\alpha$. From now on, we define $\alpha_{i j}=\alpha\left(e_{i}, e_{j}\right), i, j=1,2$.

Observe now that with the choice we made of $e_{1}$ and $e_{3}$, we can write

$$
\xi=\cos \theta e_{1}+\sin \theta e_{3} .
$$

Proposition 1. In the computations that follow, we need the following identities:

i) $\nabla_{e_{1}} e_{1}=0=\nabla_{e_{1}} e_{2}$,

ii) $d \theta\left(e_{2}\right)=0$,

iii) $\nabla \frac{\perp}{e_{2}} e_{3}=0$.

Proof. Since $\xi$ is parallel, we obtain

$$
\begin{aligned}
0= & \widetilde{\nabla}_{e_{1}} \xi=-\sin \theta d \theta\left(e_{1}\right) e_{1}+\cos \theta \nabla_{e_{1}} e_{1}+\cos \theta \alpha_{11} \\
& +\cos \theta d \theta\left(e_{1}\right) e_{3}+\sin \theta \nabla_{e_{1}}^{\perp} e_{3}-\sin \theta A_{3}\left(e_{1}\right) .
\end{aligned}
$$

The tangent and normal components of $\widetilde{\nabla}_{e_{1}} \xi$ also vanish. For the tangent component, we have

$$
-\sin \theta d \theta\left(e_{1}\right) e_{1}+\cos \theta \nabla_{e_{1}} e_{1}-\sin \theta A_{e_{3}}\left(e_{1}\right)=0 .
$$

Since $\left\{e_{1}, e_{2}\right\}$ diagonalizes $\alpha, A_{e_{3}} e_{1}=\beta e_{1} ;$ also $\nabla_{e_{1}} e_{1}=b e_{2}$. Since $e_{1}$ and $e_{2}$ are linearly independent, the vanishing of the above expression implies that both the coefficients of $e_{1}$ and $e_{2}$ vanish. Thus $\nabla_{e_{1}} e_{1}=$ $b e_{2}=0$. Since $\left\langle e_{1}, e_{2}\right\rangle=0,\left\langle\nabla_{e_{1}} e_{2}, e_{1}\right\rangle=0$, hence $\nabla_{e_{1}} e_{2}=0$.

So we have obtained (i).

By same token,

$$
\begin{gathered}
0=\widetilde{\nabla}_{e_{2}} \xi=-\sin \theta d \theta\left(e_{2}\right) e_{1}+\cos \theta \nabla_{e_{2}} e_{1}+\cos \alpha \alpha_{12} \\
+\cos \theta d \theta\left(e_{2}\right) e_{3}+\sin \theta \nabla_{e_{2}}^{\perp} e_{3}-\sin \theta A_{3}\left(e_{2}\right)
\end{gathered}
$$

whose tangent and normal components are, respectively,

$$
\begin{gathered}
\sin \theta d \theta\left(e_{2}\right) e_{1}+\cos \theta \nabla_{e_{2}} e_{1}-\sin \theta A_{3}\left(e_{2}\right)=0, \\
\cos \theta d \theta\left(e_{2}\right) e_{3}+\sin \theta \nabla_{e_{2}}^{\perp} e_{3}=0,
\end{gathered}
$$

since $\alpha_{12}=0$. Because $\nabla_{e_{2}} e_{1}=a e_{2}$ and $e_{2}$ is an eigenvector of $A_{e_{3}}$, we conclude from the first equality that $d \theta\left(e_{2}\right)=0$, hence, from the second 
equality, $\nabla_{e_{2}}^{\perp} e_{3}=0$. This proves (ii) and (iii), and concludes the proof of Proposition 1.

q.e.d.

Proposition 2. The normal vector $\alpha_{22}=\alpha\left(e_{2}, e_{2}\right)$ is parallel in the normal connection along the integral curves of $e_{2}$.

Proof. We want to show that $\nabla_{e_{2}}^{\perp} \alpha_{22}=0$. We first notice that

$$
\begin{aligned}
\nabla_{e_{2}}^{\perp} \alpha_{22} & =-\nabla_{e_{2}}^{\perp} \alpha_{11}=-\left(\nabla_{e_{2}}^{\perp} \alpha\right)\left(e_{1}, e_{1}\right)+2 \alpha\left(\nabla_{e_{2}} e_{1}, e_{1}\right) \\
& =-\left(\nabla_{e_{1}}^{\perp} \alpha\right)\left(e_{2}, e_{1}\right)+\left(\widetilde{R}\left(e_{2}, e_{1}\right) e_{1}\right)^{\perp} \\
& =-\left(\nabla_{e_{1}}^{\perp} \alpha\right)\left(e_{2}, e_{1}\right)
\end{aligned}
$$

where we have used that $H$ is parallel, $\nabla_{e_{2}} e_{1}=a e_{2}, \alpha\left(e_{1}, e_{2}\right)=0$, the Codazzi equation, and, by Eq. (1), that $\left(\widetilde{R}\left(e_{2}, e_{1}\right) e_{1}\right)^{\perp}=0$. But

$$
\left(\nabla_{e_{1}}^{\perp} \alpha\right)\left(e_{2}, e_{1}\right)=\nabla_{e_{1}}^{\perp} \alpha\left(e_{2}, e_{1}\right)-\alpha\left(\nabla_{e_{1}} e_{2}, e_{1}\right)-\alpha\left(e_{2}, \nabla_{e_{1}} e_{1}\right)=0,
$$

since $\nabla_{e_{1}} e_{1}=\nabla_{e_{1}} e_{2}=0$ (see Proposition 1 ). This proves Proposition 2.

q.e.d.

Proposition 3. $\nabla_{e_{2}} e_{2}=b e_{1}$ and $b$ is constant along the integral curves of $e_{2}$.

Proof. Since $\xi=\cos \theta e_{1}+\sin \theta e_{3}$, we have that $\left\langle\xi, e_{2}\right\rangle=0$. By differentiation, we obtain

$$
0=e_{2}\left\langle\xi, e_{2}\right\rangle=\left\langle\widetilde{\nabla}_{e_{2}} \xi, e_{2}\right\rangle+\left\langle\xi, \widetilde{\nabla}_{e_{2}} e_{2}\right\rangle .
$$

Since $\left\langle\widetilde{\nabla}_{e_{2}} \xi, e_{2}\right\rangle=0$ we have

$$
\begin{aligned}
0 & =\left\langle\widetilde{\nabla}_{e_{2}} e_{2}, \xi\right\rangle=\left\langle\nabla_{e_{2}} e_{2}, \xi\right\rangle+\left\langle\alpha\left(e_{2}, e_{2}\right), \xi\right\rangle \\
& =\left\langle\nabla_{e_{2}} e_{2}, \cos \theta e_{1}+\sin \theta e_{3}\right\rangle+\left\langle\alpha_{22}, \cos \theta e_{1}+\sin \theta e_{3}\right\rangle \\
& =\cos \theta\left\langle\nabla_{e_{2}} e_{2}, e_{1}\right\rangle+\sin \theta\left\langle\alpha_{22}, e_{3}\right\rangle .
\end{aligned}
$$

It follows that

$$
\left\langle\nabla_{e_{2}} e_{2}, e_{1}\right\rangle=b=-\operatorname{tg} \theta\left\langle\alpha_{22}, e_{3}\right\rangle .
$$

We now differentiate $b$ and obtain

$$
e_{2}(b)=\sec ^{2} \theta d \theta\left(e_{2}\right)\left\langle\alpha_{22}, e_{3}\right\rangle-\operatorname{tg} \theta\left\{\left\langle\nabla_{e_{2}}^{\perp} \alpha_{22}, e_{3}\right\rangle+\left\langle\alpha_{22}, \nabla_{e_{2}}^{\perp} e_{3}\right\rangle=0,\right.
$$

since $d \theta\left(e_{2}\right)=0$ by (ii) of Proposition $1, \nabla_{e_{2}}^{\perp} \alpha_{22}=0$ by Proposition 2, and $\nabla \frac{\perp}{e_{2}} e_{3}=0$ by (iii) of Proposition 1 . This proves Proposition 3.

q.e.d.

Now consider $E_{c}^{4} \times \mathbb{R}$ (which is our ambient space when $H$ is not an umbilical direction; see the end of the proof of Theorem 1) $\subset \mathbb{R}^{5} \times$ $\mathbb{R}$ (where $\mathbb{R}^{5}$ may possibly have a Lorentz metric) and let $\bar{\nabla}$ be the connection of $\mathbb{R}^{5} \times \mathbb{R}$.

Proposition 4. The subspace Span $\left\{e_{2}, \bar{\nabla}_{e_{2}} e_{2}\right\}$ is parallel along the integral curves of $e_{2}$. 
Proof. Notice that in this new ambient space

$$
\bar{\nabla}_{e_{2}} e_{2}=b e_{1}+\alpha_{22}+e \eta,
$$

where $\eta$ is the unit vector normal to the umbilic submanifold to $E_{c}^{4}$ in $\mathbb{R}^{5}$ which is determined by the orientation of $E_{c}^{4}$. Notice that $e$ is constant.

Now we differentiate $\bar{\nabla}_{e_{2}} \bar{\nabla}_{e_{2}} e_{2}$ and observe that:

$$
\begin{aligned}
& \bar{\nabla}_{e_{2}}\left(b e_{1}\right)=b \nabla_{e_{2}} e_{1}+b \alpha_{12}, \\
& \bar{\nabla}_{e_{2}}\left(\alpha_{22}\right)=\nabla_{e_{2}} \alpha_{22}+\nabla_{e_{2}}^{\perp} \alpha_{22}=\nabla_{e_{2}}^{\perp} \alpha_{22}-A_{\alpha_{22}} e_{2},
\end{aligned}
$$

and, by using Proposition 2, we obtain $\nabla_{e_{2}}^{\perp} \alpha_{22}=0$. Finally, since $\bar{\nabla}_{e_{2}} \eta=-e e_{2}$, we have

$$
\bar{\nabla}_{e_{1}} \bar{\nabla}_{e_{2}} e_{2}=b \nabla_{e_{2}} e_{1}+b \alpha_{12}-A_{\alpha_{22}} e_{2}-e^{2} e_{2}
$$

which is a multiple of $e_{2}$, since $\alpha_{12}=0, \nabla_{e_{2}} e_{1}=-b e_{2}$, and for all $v$ normal $A_{v} e_{2}$ is a multiple of $e_{2}$. This proves Proposition $4 . \quad$ q.e.d.

Proposition 5. The integral curves of $e_{2}$ are plane circles.

Proof. First, notice that $\left|\bar{\nabla}_{e_{2}} e_{2}\right|$ is constant. For, $b e_{1}, \alpha_{22}$, and $e \eta$ are orthogonal vectors which have constant norms (recall that $\alpha_{22}$ is parallel along the integral curves of $\left.e_{2}\right)$. Since $\bar{\nabla}_{e_{2}} \bar{\nabla}_{e_{2}} e_{2}$ is a multiple of $e_{2}$, we conclude the proof of Proposition 5.

q.e.d.

Proposition 6. The integral curves of $e_{2}$ lie in parallel planes.

Proof. We first observe that the integral curves of $e_{2}$ are the level curves of the height function $h(p)=\langle x(p), \xi\rangle, p \in M$. To see that let $w_{1}$ and $w_{2}$ be first order differential forms defined in $M$ by $w_{i}\left(e_{j}\right)=\delta_{i j}$, $i, j=1,2$. Then

$$
d h=\langle d x, \xi\rangle=\left\langle w_{1} e_{1}+w_{2} e_{2}, \xi\right\rangle,
$$

hence

$$
d h\left(e_{2}\right)=\left\langle w_{1}\left(e_{2}\right) e_{1}+w_{2}\left(e_{2}\right) e_{2}, \xi\right\rangle .
$$

Since $\xi=e_{1} \cos \theta+e_{3} \sin \theta$, we conclude that

$$
d h\left(e_{2}\right)=\left\langle e_{2}, \xi\right\rangle=0,
$$

which implies that the height function is constant along the integral curves of $e_{2}$.

Given a point in a given integral curve of $e_{2}$, any other such integral curve, close enough to the given one, can be connected to this point by a unique gradient line; this allows us to talk about corresponding points at distinct integral curves of $e_{2}$ that are close enough.

Notice that the gradient lines parametrized by arclength are integral curves of the vector field $e_{1}$. Since,

$$
\bar{\nabla}_{e_{1}} e_{2}=\nabla_{e_{1}} e_{2}+\alpha_{12}=0,
$$


we see that the tangent lines at corresponding points of distinct integral curves of $e_{2}$, that are close enough, are parallel. Thus such curves are in parallel planes.

Since the critical points of the height function are the zeroes of the analytic differential $d h_{p}: X_{p} \rightarrow\left\langle X_{p} . \xi\right\rangle$, where $X_{p}$ is a tangent vector at $p$, they form a closed set $F$ in $M$ with no interior points; notice that we have discarded Case a, so we cannot have $\left\langle X_{p}, \xi\right\rangle \equiv 0$. The complementary set $\mathcal{C} F$ is an open and dense set that may have a number of connected components. Clearly, for a given connected component of $\mathcal{C} F$, the planes containing the trajectories of $e_{2}$ are all parallel to a fixed plane.

Now, given a point that belongs to the boundary of two connected components $(\mathcal{C} F)^{1}$ and $(\mathcal{C} F)^{2}$, consider the two planes $P_{1}$ and $P_{2}$ that are limit of the planes containing points of the sequences $\left.\left\{p_{i}^{1}\right\} \in \mathcal{C} F\right)^{1}$ and $\left.\left\{p_{1}^{2}\right\} \in \mathcal{C} F\right)^{2}$, respectively, that converge to $p$. We claim that $P_{1}=$ $P_{2}$. Otherwise, their intersections would be a straight line that, by completeness extend to the whole $M$. This implies that $K \equiv 0$, a contradiction.

It follows that all planes containing the integral curves of $e_{2}$ are parallel to a fixed plane, and this proves Proposition $6 . \quad$ q.e.d.

Remark 6. In the present situation, where $M$ is homeomorphic to a sphere, a simpler argument would prove Proposition 6. This Proposition, however, will be used in the proof of Theorem 3, and there we will need the full force of the above argument.

Now, decompose $\mathbb{R}^{5} \times \mathbb{R}$ by two orthogonal subspaces $P \oplus P^{\perp}$, where $P$ is a plane which contains one integral curve of $e_{2}$, and $P^{\perp}$ is its orthogonal complement. The integral curve of $e_{2}$ in $P$ can be parametrized by

$$
\hat{g}+r \cos \theta f_{1}+r \sin \theta f_{2},
$$

where $\left\{f_{1}, f_{2}\right\}$ is an orthonormal basis of $P, r$ is a function on $M$ such that $e_{2}(r)=0$, and $\hat{g} \in P$ is the position vector of the center of the circle in (5).

As we move along a gradient line, the level curves of the height function $h$ (circles in planes parallel to $P$ ) are given by $\beta(s)=\hat{g}(s)+$ $r(s) \cos \theta f_{1}+r(s) \sin \theta f_{2}$. Project the curve $\beta(s)$ into $P$ and keep, for simplicity, the same notation as in the above equality.

We claim that, as we move along a gradient line, the point $\hat{g}(s)$ remains fixed, i.e., $\hat{g}^{\prime}(s)=0$.

To see that, let $\hat{e}_{1}$ be the projection of $e_{1}$ into $P$. Then

$$
\hat{e}_{1}=\frac{\partial}{\partial s} \beta(s)=\hat{g}^{\prime}(s)+r^{\prime}(s) \cos \theta f_{1}+r^{\prime}(s) \sin \theta f_{2} .
$$


Since $e_{2} \in P,\left\langle\hat{e}_{1}, e_{2}\right\rangle=0$. By observing that $e_{2}=-\sin \theta f_{1}+\cos \theta f_{2}$, we obtain that

$$
0=\left\langle\hat{e}_{1}, e_{2}\right\rangle=\left\langle\hat{g}^{\prime}(s), e_{2}\right\rangle,
$$

and, since $e_{2}$ spans $P$, we conclude that $\hat{g}^{\prime}(s) \equiv 0$, as we claimed.

We have shown that if we are not in Case (a) above, item (4) of Theorem 2 holds. By using the analyticity of the immersion in the way as we did before (cf. Remark 4), we will show that item (4) of Theorem 2 holds in $M$.

As we have seen before, if $H$ is an umbilical direction everywhere, $x(M) \subset E_{c}^{n}$. In this case, we can use Yau's theorem mentioned in Section 2 and conclude the other items of Theorem 2 .

\section{Proof of Theorem 3}

Consider the complex structure on $M$ given by $z=u+i v$, where $(u, v)$ are isothermal parameters in which the metric on $M$ is given by $d s^{2}=\lambda^{2}\left(d u^{2}+d v^{2}\right)$. Notice that $Q^{(2,0)}=\psi d z^{2}$, where $\psi$ a complex function that, from our hypothesis, is holomorphic. Furthermore, since $|Z|=\lambda$,

$$
\left|Q^{(2,0)}\right|=\left|Q^{(2,0)}\left(\frac{Z}{\lambda}, \frac{Z}{\lambda}\right)\right|=\frac{1}{\lambda^{2}}\left|Q^{(2,0)}(Z, Z)\right|=\frac{1}{\lambda^{2}}|\psi| .
$$

Now, if $Q^{(2,0)}$ is not identically zero, its zeroes are isolated. We claim that away from the zeroes of $Q^{(2,0)}$

$$
\Delta \log \left|Q^{(2,0)}\right|=4 K \text {. }
$$

Indeed, since $Q^{(2,0)}$ is holomorphic, $\log \left(\left|Q^{(2,0)}\right| \lambda^{2}\right)$ is harmonic. Thus,

$$
\Delta \log \left|Q^{(2,0)}\right|=-2 \Delta \log \lambda=4 K,
$$

where in the last equality, we used the expression of the Gaussian curvature in isothermal coordinates. This proves our claim.

Since $K \geq 0, M$ is parabolic by Huber's theorem ([7]); this means that all subharmonic functions bounded from above are constants. We will show that $\left|Q^{(2,0)}\right|$ is bounded. Thus $\left|Q^{(2,0)}\right|=$ constant, hence $K \equiv 0$ in $M$.

First, notice that, since

$$
Q(X, Y)=2\langle\alpha(X, Y), H\rangle-c\langle X, \xi\rangle\langle Y, \xi\rangle,
$$

by letting $\beta(X, Y)=\langle X, \xi\rangle\langle Y, \xi\rangle$, we obtain

$$
\left|Q^{(2,0)}\right|^{2} \leq\left|2\left\langle\alpha^{(2,0)}, H\right\rangle\right|^{2}+c^{2}\left|\beta^{(2,0)}\right|^{2} .
$$

But $\left|\beta^{(2,0)}\right| \leq 1$, and a simple computation shows

$$
\left\langle\alpha^{(2,0)}, H\right\rangle^{2}=\left(\text { trace of } A_{H}\right)^{2}-\operatorname{det}\left(A_{H}\right) .
$$


Now choose a frame $\left(e_{1}, e_{2}, f_{1}, f_{2}, f_{3}\right)$ where $e_{1}, e_{2}$ are orthonormal tangent vectors, and $f_{1}, f_{2}, f_{3}$ are orthonormal normal vectors, such that $f_{1}=H /|H|$. Notice that, since the mean curvature vector $H$ has no component along $f_{2}$ and $f_{3}$, we have that $\left(\operatorname{trace} A_{f_{i}}\right)=0$, for $i \neq 1$. Also, since $H$ is parallel, (trace $\left.A_{H}\right)^{2}$ is constant. Thus, it suffices to show that $\left(\operatorname{det} A_{H}\right) \geq 0$ to conclude from (6) that $\left\langle\alpha^{(2,0)}, H\right\rangle^{2}$ is bounded.

For that, observe that, by hypothesis, $\left\langle\widetilde{R}\left(e_{1}, e_{2}\right), e_{1}, e_{2}\right\rangle \leq 0$. Since,

$$
K=\left\langle\widetilde{R}\left(e_{1}, e_{2}\right) e_{1}, e_{2}\right\rangle+\sum_{i=1}^{3} \operatorname{det} A_{f_{i}}
$$

and $K \geq 0$, we have that $\sum_{i=1}^{3} \operatorname{det} A_{f_{i}} \geq 0$. Since, for $i \neq 1, A_{f_{i}}$ is symmetric and has trace zero, then $\operatorname{det}\left(A_{f_{i}}\right) \leq 0$, if $i \neq 1$. Thus, by using (7), we conclude finally that $\left(\operatorname{det} A_{H}\right) \geq 0$, hence $\left\langle\alpha^{(2,0)}, H\right\rangle$ is bounded and the same holds for $\left|Q^{(2,0)}\right|$.

Thus, we have shown that if $Q^{(2,0)}$ is not identically zero, then $K \equiv 0$, which proves (1) of Theorem 3.

If $Q^{(2,0)} \equiv 0$, we first observe that Case (a) and (b) can occur. By using analyticity in the same way we did before in this paper we can easily check that Case (a) leads to items (2) and (3) whereas Case (b) leads to the fact that $x(M) \subset E_{c}^{2} \times \mathbb{R}$; this is already contained in item (4). With these cases out of the way, we can choose an appropriate frame and proceed as in Theorem 2 except that the level curves of the height function are not necessarily circles. This proves completely item 4 and concludes the proof of Theorem 3 .

\section{References}

[1] U. Abresch \& H. Rosenberg, A Hopf differential for constant means curvature surfaces in $S^{2} \times \mathbb{R}$ and $H^{2} \times \mathbb{R}$, Acta Math. 193 (2004) 141-174, MR 2134864, Zbl 1078.53053 .

[2] U. Abresch \& H. Rosenberg, Generalized Hopf differentials, Matemtica Contempornea 28 (2005), Sociedade Brasileira de Matemtica, 1-28, MR 2195187, Zbl 1118.53036.

[3] H. Alencar, M. do Carmo \& R. Tribuzy, A theorem of Hopf and the CauchyRiemann inequality, Communications in Analysis and Geometry 15 (2007) 283298, MR 2344324, Zbl 1134.53031.

[4] S.S. Chern, On surfaces of constant mean curvature in a three-dimensional space of constant curvature, Geometric Dynamics, Springer Lecture Notes, 1007, 1983, 104-108, MR 0730266, Zbl 0521.53006. 
[5] J.H. Eschenburg \& R. Tribuzy, Existence and uniqueness of maps into affine homogeneous spaces, Rend. Sem. Mat. Univ. Padova, 89 (1993), 11-18, MR 1229038, Zbl 0803.53032.

[6] H. Hopf, Differential Geometry in the large, Lectures Notes in Math., 1000, Springer-Verlag, 1983, MR 0707850, Zbl 0669.53001.

[7] A. Huber, On subharmonic functions and differential geometry in the large, Comm. Math. Helv. 32 (1957) 13-71, MR 0094452, Zbl 0080.15001.

[8] S.T. Yau, Submanifolds with constant mean curvature I, American Journal of Mathematics 96 (1974) 346-366, MR 0370443, Zbl 0304.53041.

Universidade Federal de Alagoas Instituto DE MATEMÁtica 57072-900 MACEIÓ, AL, BRASIL

E-mail address: hilario@mat.ufal.br

IMPA

Estrada D. Castorina, 110 22460-320 Rio de Janeiro, RJ, Brasil E-mail address: manfredo@impa.br

Universidade Federal do Amazonas Instituto DE CiÊnCias Exatas Departamento de Matemática Av. Rodrigo Otávio Jordão Ramos, 3000 63077-000 Manaus, AM, Brasil E-mail address: tribuzy@pesquisador.cnpq.br 